\title{
Redescription of Gessyella latridopsis (Johnston et Mawson, 1945) (Nematoda: Capillariidae), a parasite of latrid fishes from the Southern Hemisphere
}

\author{
María Alejandra Rossin and Juan Tomás Timi
}

Laboratorio de Parasitología, Departamento de Biología, Facultad de Ciencias Exactas y Naturales, Universidad Nacional de Mar del Plata - Consejo Nacional de Investigaciones Científicas y Técnicas (CONICET), Funes 3350, (7600) Mar del Plata, Argentina

\begin{abstract}
The type species of the monotypic genus Gessyella Freitas, 1959, G. latridopsis (Johnston et Mawson, 1945) (Nematoda: Capillariidae), is redescribed from specimens found in the rectum of the marine fish Nemadactylus bergi Norman (Latridae, Perciformes) (a new host record) from the southwestern Atlantic Ocean off Mar del Plata, Argentina. In addition to the morphological and biometrical variability of this species, some new, previously unreported taxonomic features, such as the extent of the spination of the spicular sheath, the morphology of the spicular canal, the distal end of the spicule, the ejaculatory duct, the cloaca, the seminal vesicle, vas deferens and the testis, are described for the first time. Despite the long geographical distance of G. latridopsis records (Australia vs. Argentina), the morphology of the newly collected specimens is in agreement with the existing descriptions of $G$. latridopsis and both host species are closely related. Therefore, the Argentine specimens are considered to belong to this species.
\end{abstract}

Key words: parasitic nematodes, marine fish, Argentine Sea, Latridae, Gessyella

The genus Gessyella Freitas, 1959 was erected by Freitas (1959) to accommodate specimens described as Capillaria latridopsis Johnston et Mawson, 1945 from Latridopsis forsteri Castelnau (Latridae) from Australia (Johnston and Mawson 1945). The original description of G. latridopsis was based on two incomplete specimens, one male and one female. This genus was characterised by the presence of lateral caudal alae in males and the presence of eggs enclosed in special capsules (Freitas 1959). Later, in view of the inadequate original species description and too schematic drawings, Moravec (1987) re-examined the type specimens; however, only the incomplete type male (a part of its oesophageal region was missing) was available, whereas the type female was found to be lost. In spite of the poor condition of the specimen, Moravec (1987) redescribed the male and confirmed the validity of the independent genus Gessyella. He considered the presence of caudal lateral alae in male a unique feature among fish capillariids; however, the structure of eggs could not be confirmed. As far as we know, representatives of this genus have not been recorded since the original description of $C$. latridopsis by Johnston and Mawson (1945).

At present, 14 genera of the family Capillariidae containing species parasitizing cold-blooded vertebrates are recognized (Moravec 2001). In a series of recent papers, representatives of four of these genera have been described from fishes inhabiting the Argentine Sea, namely Capillaria (Procapillaria) navoneae Timi, Rossin et Lanfranchi, 2006, an intestinal parasite of the conger Conger orbignianus Valenciennes, Paracapillaria (Paracapillaria) argentinensis Timi, Rossin, Lanfranchi et Etchegoin, 2007, parasitic in the stomach of the sandperch Pinguipes brasilianus Cuvier, Paracapillaroides acanthocotylus Rossin et Timi, 2009, a parasite of the stomach of $\mathrm{Ne}$ madactylus bergi (Norman), and Capillostrongyloides congiopodi Cantatore, Rossin, Lanfranchi et Timi, 2009, a parasite of the gall bladder of Congiopodus peruvianus Cuvier et Valenciennes (see Timi et al. 2006, 2007, Cantatore et al. 2009, Rossin and Timi 2009).

During a parasitological survey carried out on additional specimens of $N$. bergi landed by fishermen at Mar del Plata port, Argentina, capillariid nematodes referable to Gessyella latridopsis were found in the rectum of these fish. A redescription of this hitherto little-known species is given based on the newly collected specimens.

\section{MATERIALS AND METHODS}

A total of 100 specimens of Nemadactylus bergi caught by commercial trawlers operating at the Mar del Plata port $\left(38^{\circ} 08^{\prime} \mathrm{S}\right.$, $57^{\circ} 32^{\prime} \mathrm{W}$ ), during May to June 2008, were examined for nematodes immediately after capture. Fish were dissected and the 
intestines were removed and examined under a stereoscopic microscope. A total of 28 capillariid nematodes (19 complete and 9 incomplete specimens) were collected. The nematodes were fixed in $4 \%$ formaldehyde solution, preserved in $70 \%$ ethanol and then studied and measured by light microscopy. Drawings were made using a drawing tube. All measurements are given in micrometres unless otherwise indicated; the mean is followed by the range in parentheses. The material studied was deposited in the Colección Helmintológica del Museo de La Plata (C.H.M.L.P.), La Plata, Argentina and in the Helminthological Collection of the Institute of Parasitology, Biology Centre of ASCR in České Budějovice, Czech Republic.

\section{RESULTS}

Gessyella latridopsis (Johnston et Mawson, 1945) Freitas, 1959

General: Medium-sized nematodes; males smaller than females. Cephalic end narrow and rounded with a constriction near end. Cephalic papillae indistinct. $\mathrm{Cu}-$ ticle smooth. Two lateral bacillary bands extending along whole body. Nerve ring not located, difficult to see. Muscular oesophagus-stichosome junction oblique. Stichosome consisting of 33-44 stichocytes arranged in single row, each bearing medium-sized, regular, central nucleus. Stichocytes differing in size, 60-190 long, each subdivided in approximately 9-12 annuli. Two small glandular cells present at oesophago-intestinal junction.

Male (9 specimens): Body 8.0 (6.9-8.9) mm long, maximum width 72 (62-88); width at oesophago-intestinal junction 65 (55-75). Bacillary bands 17 (13-20) wide at mid-region of body. Muscular oesophagus 277 (237-312) long; stichosome 4.8 (3.6-5.3) mm long, composed of 40 (38-44) stichocytes; entire oesophagus 4.9 (3.6-5.6) $\mathrm{mm}$ long, representing 66 (58-72)\% of body length. Length forebody/hindbody ratio $1: 2$. Testis reaches anteriorly to level of oesophago-intestinal junction. Seminal vesicle not differentiated from vas deferens and testis, length of seminal vesicle and vas deferens 1.7 (1.1-2.5) mm. Ejaculatory duct elongate, 377 (275-475) long. Intestine joins cloaca immediately posterior to ejaculatory duct. Cloaca 608 (512-712) long, representing 8 (7-8)\% of body length; anterior cloaca 300 (250-362) long, posterior cloaca 307 (250-375) long. Spicule inside spicular canal, of regular width and divided into two parts; anterior part well sclerotized and with dense surface transverse grooves, distal part weakly sclerotized with pointed distal tip. Spicule total length 371 (330-400), anterior sclerotized part 308 (262-362) long and distal part 60 (50-65) long. Spicule, representing $4(3-4) \%$ of body length, 8 (6-9) wide at proximal end and $9(7-10)$ wide at middle portion. Spicular canal 138 (100-192) long. Spicular sheath fully invaginated in majority of specimens, but showing complex structure in those with evaginated spicular sheath, comprising short and cylindrical proximal part without spines, and globular middle portion fully covered by minute spinules. Distal end of spicular sheath could not be observed. Caudal cuticle laterally expanded, forming irregular, narrow lateral alae distinctly separated from caudal bursa and starting at 182 (155-207) from caudal end. Posterior end of body obtuse, bearing welldeveloped cuticular membranous bursa supported by two lateral rays and one pair of large lateral papillae present at base of caudal projections. Width of body just in front of bursa 46 (30-75), length of membranous bursa 9 (7-12). Cloacal opening terminal.

Female (8 specimens): Body 10 (5-13) mm long, maximum width 73 (55-100). Bacillary bands 32 (2555) wide. Muscular oesophagus 306 (250-337) long; stichosome 5 (3-7) mm long, composed of 37 (33-39) stichocytes; entire oesophagus 5 (3-7) mm long, representing 56 (52-63)\% of body length. Length forebody/ hindbody ratio $1: 1.3$. Cuticular expansion present anterior to vulva. Vulva situated just posterior to oesophago-intestinal junction. Muscular vagina directed posteriorly from vulva, maximum length and width 218 and 37, respectively. Eggs numerous, elongate with protruding polar plugs and uncleaved content, 60 (57-62) long by 26 (25-27) wide; polar plugs 5 (3-6) long by 5 (5-7) wide; egg wall two-layered; inner hyaline layer thin, outer layer thicker and darker; mature eggs enclosed in irregular transparent capsules. Ovary extending posteriorly to approximately distal end of intestine, 81 (28-200) from caudal end. Rectum 101 (50-137) long. Caudal end rounded; anus subterminal. Tail 7 (5-10) long.

Ho st: Nemadactylus bergi Norman (Latridae, Perciformes).

Site of infection: Rectum.

L o c a lity: SW Atlantic off Mar del Plata, $38^{\circ} 08^{\prime} \mathrm{S}, 57^{\circ} 32^{\prime} \mathrm{W}$, Buenos Aires Province, Argentina.

Date of collection: May-June, 2008.

Vouchers: C.H.M.L.P Coll. No. 5951; Helminthological

Collection of the Institute of Parasitology, Biology Centre,

ASCR, České Budějovice (Coll. No. N-931).

Prevalence: $13 / 100(13 \%)$.

Mean intensity (range): $2.1(1-4)$.

\section{DISCUSSION}

The present study constitutes the first complete morphological description of the type species of Gessyella, providing also the first set of data on its biometrical variability. Most of the morphological characters observed in the present material from $N$. bergi agree with the diagnostic generic characteristics (Freitas 1959, Moravec 1987, 2001): the stichosome consisting of a single row of stichocytes; lateral caudal alae in the male present; the posterior end of male with the membranous bursa supported by two large, lobe-like lateral papillae and two elongate dorsolateral projections with their distal ends exceeding the rim of the bursa, and surface of the spicule with numerous transverse grooves. The specimens herein 

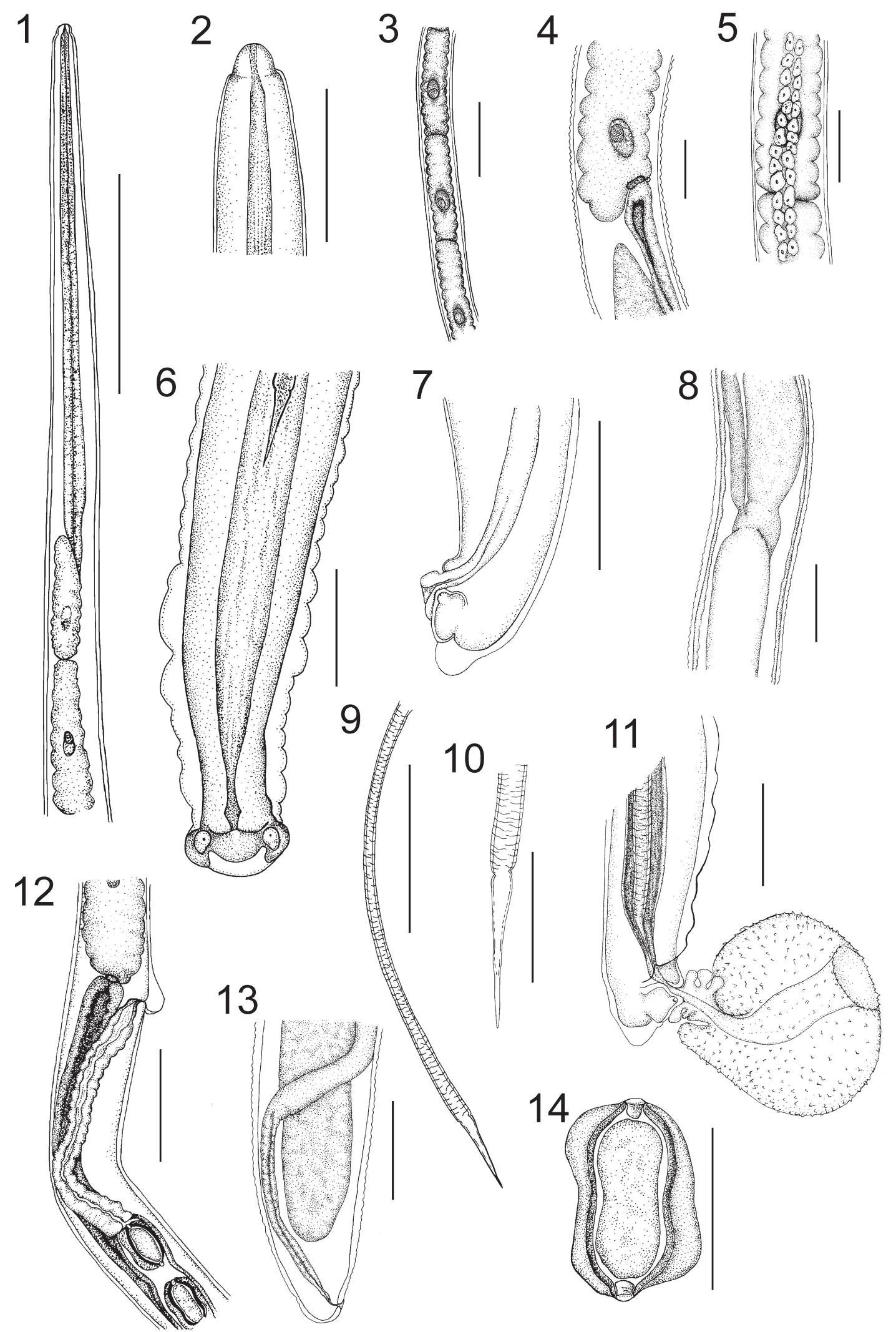

Figs. 1-14. Gessyella latridopsis (Johnston et Mawson, 1945) Freitas, 1959. Fig. 1. Anterior end. Fig. 2. Detail of anterior end. Fig. 3. Stichosome. Fig. 4. Oesophago-intestinal junction. Fig. 5. Bacillary band at level of stichosome. Fig. 6. Male posterior end, ventral view. Fig. 7. Male posterior end, lateral view. Fig. 8. Intestine-ejaculatory duct junction. Fig. 9. Spicule. Fig. 10. Distal end of spicule. Fig. 11. Partially evaginated spicular sheath, lateral view. Fig. 12. Female, region of vulva, lateral view. Fig. 13. Female tail, lateral view. Fig. 14. Egg. Scale bars: Figs. 1, $9=125 \mu \mathrm{m}$; Figs. 2-8, 10-14=50 $\mu \mathrm{m}$. 

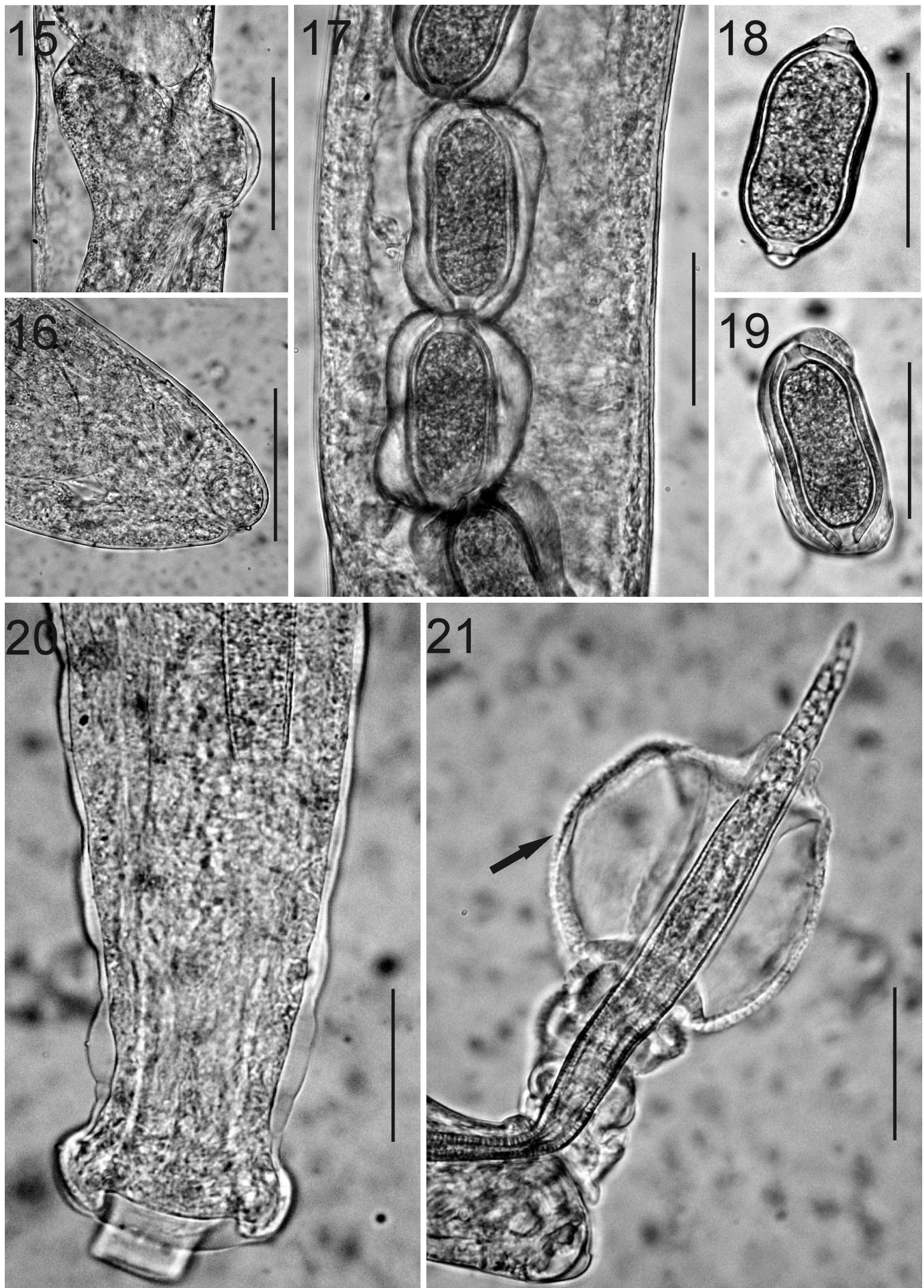

Figs. 15-21. Gessyella latridopsis (Johnston et Mawson, 1945) Freitas, 1959. Fig. 15. Female, region of vulva, lateral view. Fig. 16. Female tail, lateral view. Fig. 17. Eggs in uterus. Fig. 18. Young egg. Fig. 19. Mature egg. Fig. 20. Male, posterior end, dorsal view. Fig. 21. Male, evaginated spicule and spicular sheath (lateral view), arrow - spinules covering globose middle portion of spicular sheath. Scale bars $=50 \mu \mathrm{m}$. 
described showed a spicular sheath devoid of spines at its basal portion, instead of spiny as described originally. Only two males had the spicular sheath evaginated, allowing the description of its morphology and ornamentation. It is probable that the invaginated spicular sheath of the lectotype designated by Moravec (1987) and its poor preservation condition had not allowed a detailed observation of this structure.

On the other hand, the general morphology and all morphometric features of specimens from Argentina agree with those described for $G$. latridopsis from Australia (Moravec 2001), and there is no reason for considering them as a distinct species, unless a complete description of G. latridopsis from the type host and locality is available. Some features provided for the first time for this species, such as the spination of the spicular sheath and the morphology of the spicular canal, the distal end of the spicule, the ejaculatory duct, the cloaca, the seminal vesicle and vas deferens and the testis, will allow confirming or rejecting the hypothesis that parasites from Australia and Argentina are conspecific.

This nematode has not been recorded since it was described by Johnston and Mawson (1945) as a parasite of the trumpeter Latridopsis forsteri in the Indian Ocean, near the coast of South Australia (Kangaroo Island). Its presence in distant geographic regions, i.e., South America (Atlantic Ocean) and Australia (Indian Ocean), can be explained by the hosts' phylogenetic relation and similar habits. In fact, $N$. bergi, although it has been largely reported as a member of the cheilodactylid genus Cheilodactylus Lacépède (see Greenwood 1995), actually belongs to Latridae, the same family to which Latridopsis forsteri belongs (Burridge 1999, Burridge and Smolenski 2004). Furthermore, members of this fish family occur in subtropical to cool-temperate waters throughout the Southern Hemisphere (Burridge 1999) and display similar feeding habits, preying on small benthic invertebrates (Burridge and Smolenski 2004).

Acknowledgements. The authors wish to thank Dr. František Moravec (Institute of Parasitology, Biology Centre of the Academy of Sciences of the Czech Republic, České Budějovice) for his useful review of an early version of the manuscript and Mr. Roberto Mazella and Mr. Hugo Mazella from the fish market Albatros, Mar del Plata, for kindly providing fish samples. The present study was funded by grants from CONICET (PIP No. 112-200801-0024), ANPCYT (PICT No. 02199) and Universidad Nacional de Mar del Plata (EXA 442/08).

\section{REFERENCES}

BurRidge C.P. 1999: Molecular phylogeny of Nemadactylus and Acantholatris (Perciformes: Cirrhitoidea: Cheilodactylidae), with implications for taxonomy and biogeography. Mol. Phylogenet. Evol. 13: 93-109.

Burridge C.P., Smolenski A.J. 2004: Molecular phylogeny of the Cheilodactylidae and Latridae (Perciformes: Cirrhitoidea) with notes on taxonomy and biogeography. Mol. Phylogenet. Evol. 30: 118-127.

Cantatore D., Rossin M.A., Lanfranchi A.L., Timi J.T. 2009: A new species of Capillostrongyloides (Nematoda: Capillariidae) parasitizing Nemadactylus bergi (Perciformes: Cheilodactylidae) from the Argentine Sea. J. Parasitol. 95: 388-391.

Freitas J.F.T. 1959: Esboco de novo arranjo sistemático para os Nematodos Capilariíneos (Trichuroidea). Atas Soc. Biol. Rio J. 3: 4-6.

Greenwood P.H. 1995: A revised familial classification for certain cirrhitoid genera (Teleostei, Percoidei, Cirrhitoidea), with comments on the group's monophyly and taxonomic ranking. Bull. Nat. Hist. Mus. Lond. 61: 1-10.
Johnston T.H., Mawson P.M. 1945: Capillariid nematodes from South Australian fish and birds. Trans. R. Soc. S. Aust. 69: 243248.

Moravec F. 1987: Revision of capillariid nematodes (subfamily Capillariinae) parasitic in fishes. Studie ČSAV No. 3, Academia, Praha, 141 pp.

Moravec F. 2001: Trichinelloid Nematodes Parasitic in Coldblooded Vertebrates. Academia, Prague, 429 pp.

Rossin M.A., Timi J.T. 2009: A new species of Paracapillaroides (Nematoda: Capillariidae) parasitizing Nemadactylus bergi (Perciformes: Latridae) from the Argentine Sea. Folia Parasitol. 56: 37-40.

Timi J.T., Rossin M.A., Lanfranchi A.L. 2006: A new species of Capillaria (Nematoda: Capillariidae) parasitizing Conger orbignianus (Pisces: Congridae) from Argentina. J. Parasitol. 92: 628-630.

Timi J.T., Rossin M.A., Lanfranchi A.L., Etchegoin J.A. 2007: A new species of Paracapillaria (Nematoda: Capillariidae) parasitizing the Brazilian sandperch Pinguipes brasilianus (Pisces: Pinguipedidae) from Argentina. J. Parasitol. 93: 922-924. 